\title{
Advances in EBSD sample preparation by broad ion beam milling
}

\author{
Laurie Palasse $^{1}$ and Pawel Nowakowski ${ }^{2}$
}

${ }^{1}$ Bruker Nano Analytics, Berlin, Berlin, Germany, ${ }^{2}$ E.A. Fischione Instruments, Inc., United States

Specimen preparation for successful electron backscatter diffraction (EBSD) analyses is a complex and multistage process. Because the EBSD signal comes from the top few nanometers of the sample, it requires high-quality surface preparation. The surface must be free from crystal lattice damage and plastic deformation, as well as from contamination and oxidation.

This makes the use of mechanical polishing technique limited to relatively coarse-grained specimens containing phases of similar hardness. Polyphase samples comprising soft and hard materials are extremely difficult to polish with this technique. For example, if one phase is hard and brittle and the other phase is soft and ductile, the rate of material removal can vary widely. In addition, hard particles detached during grinding and coarse polishing phases will act as a grinding medium and tear the soft matrix of the second phase during the finer polishing steps. The result is a surface inappropriate for EBSD analyses.

Electrochemical polishing is another sample preparation technique. It is fast and can be automated, but its application is limited to conductive and monophase materials with homogenous surfaces. This method can be hazardous because it uses highly acidic and corrosive solutions.

Focused ion beam (FIB) milling can also be used for EBSD sample preparation; however, it has limitations. The preparation area is small, below $250 \mu \mathrm{m}^{2}$, and in some cases, FIB milling causes material damage that must be removed prior to EBSD acquisition [1-3].

The alternative specimen preparation technique for coarse to fine-grained and mono- to multiphase materials is broad ion beam (BIB) milling.

BIB milling achieves high-quality polished surfaces on almost any material without the need for chemicals. It removes residual artifacts incurred during mechanical cutting, grinding, and polishing steps. The advantages of BIB milling over FIB milling is the ability to prepare large sample areas of up to 50 $\mathrm{mm}$ in diameter [Model 1062 TrionMill, Fischione Instruments], but also to produce samples suitable for high-speed EBSD acquisition without requiring subsequent damage removal steps. The large area milling capability is a combination of milling using three ion sources simultaneously, wide ion beam size, and adjustable beam position. The flexibility in beam size and position allows milling parameter optimization for specific applications and sample sizes. These characteristics are applicable to multiphase materials with significant differences in hardness and milling rates (Figure 1).

BIB milling is the ideal sample preparation method for EBSD measurement of multi-layers because it can prepare large (millimeter scale) cross-section samples from fragile and / or brittle materials. It can also produce larger (centimeter scale) pristine surfaces suitable for high resolution EBSD mapping [4]. And it prevents preparation-induced phase transformation of metastable phases, for example, austenite-tomartensite dynamic transformation in steels [5, 6]. Use of a cryo stage allows BIB milling of beamsensitive materials, such as Zn or low temperature solder junctions, as shown in Figure 1. Cryogenic BIB milling, combined with vacuum/inert gas transfer, permits sample preparation of Li-ion battery materials $[7,8]$. 
Additionally, BIB can be applied after high removal rate techniques, like lasers and plasma ion beams, as final polishing for 2D or 3D EBSD measurement.

In this presentation, we review EBSD results acquired with $e$-Flash ${ }^{\mathrm{FS}}$ EBSD detector [Bruker Nano Analytics] from various materials prepared by mechanical polishing and by BIB milling. Sample types include steel, titanium, zirconium alloys, and solder bumps. EBSD analyses show that if there is surface topography produced by BIB milling, it has no effect on EBSD results. As shown in Figure 2, EBSD results show no correlation between surface topography and the distribution of crystallographic orientation and misorientations (Figure 2). That confirms that BIB milling is an excellent surface preparation technique for high quality EBSD measurements.

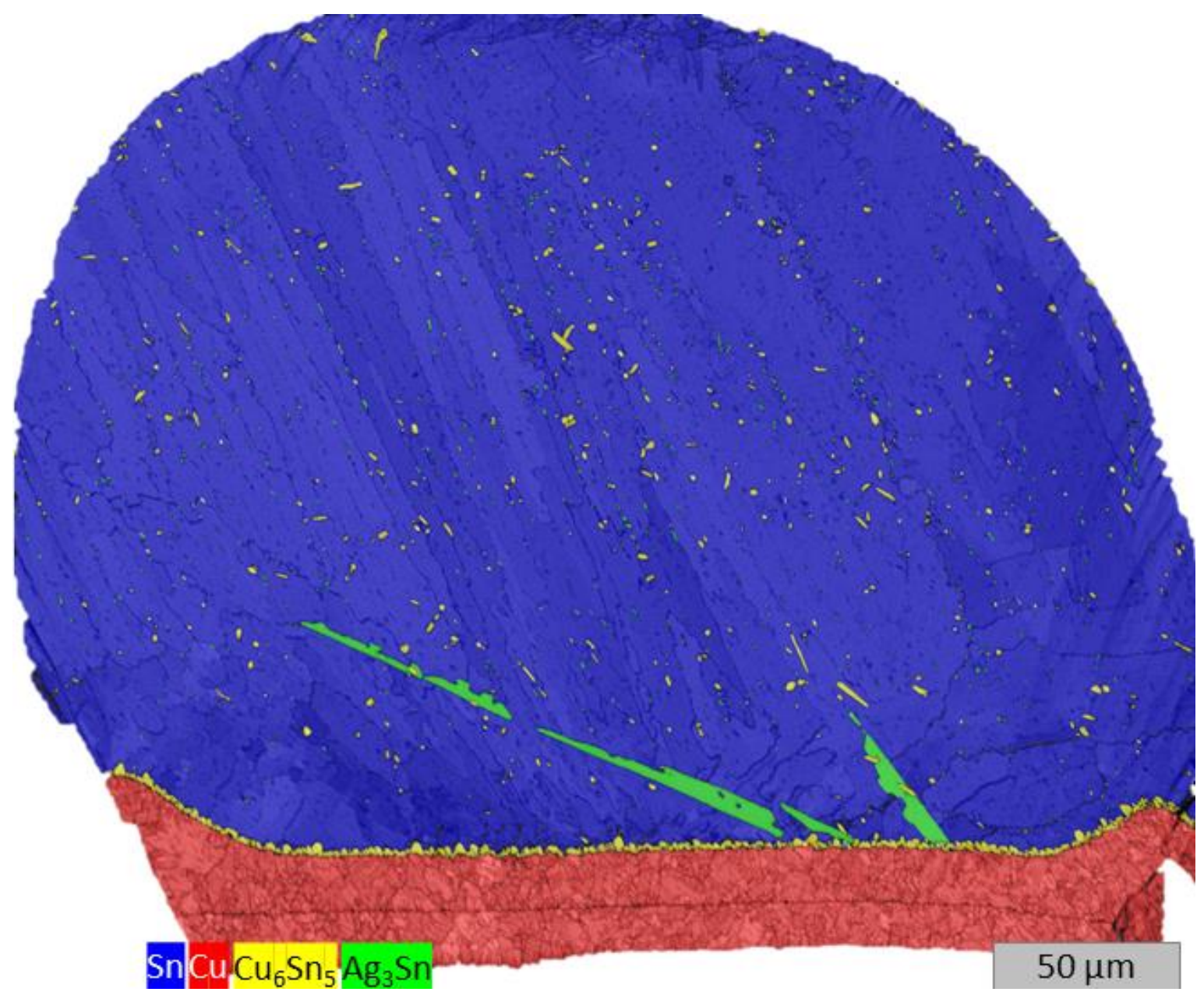

Figure 1. EBSD phase map of a Sn-Cu solder bump prepared under cryogenic conditions. Measurement was performed with e-FlashFS EBSD detector [Bruker Nano Analytics]; neither sample coating nor low vacuum conditions were required for the measurement. 

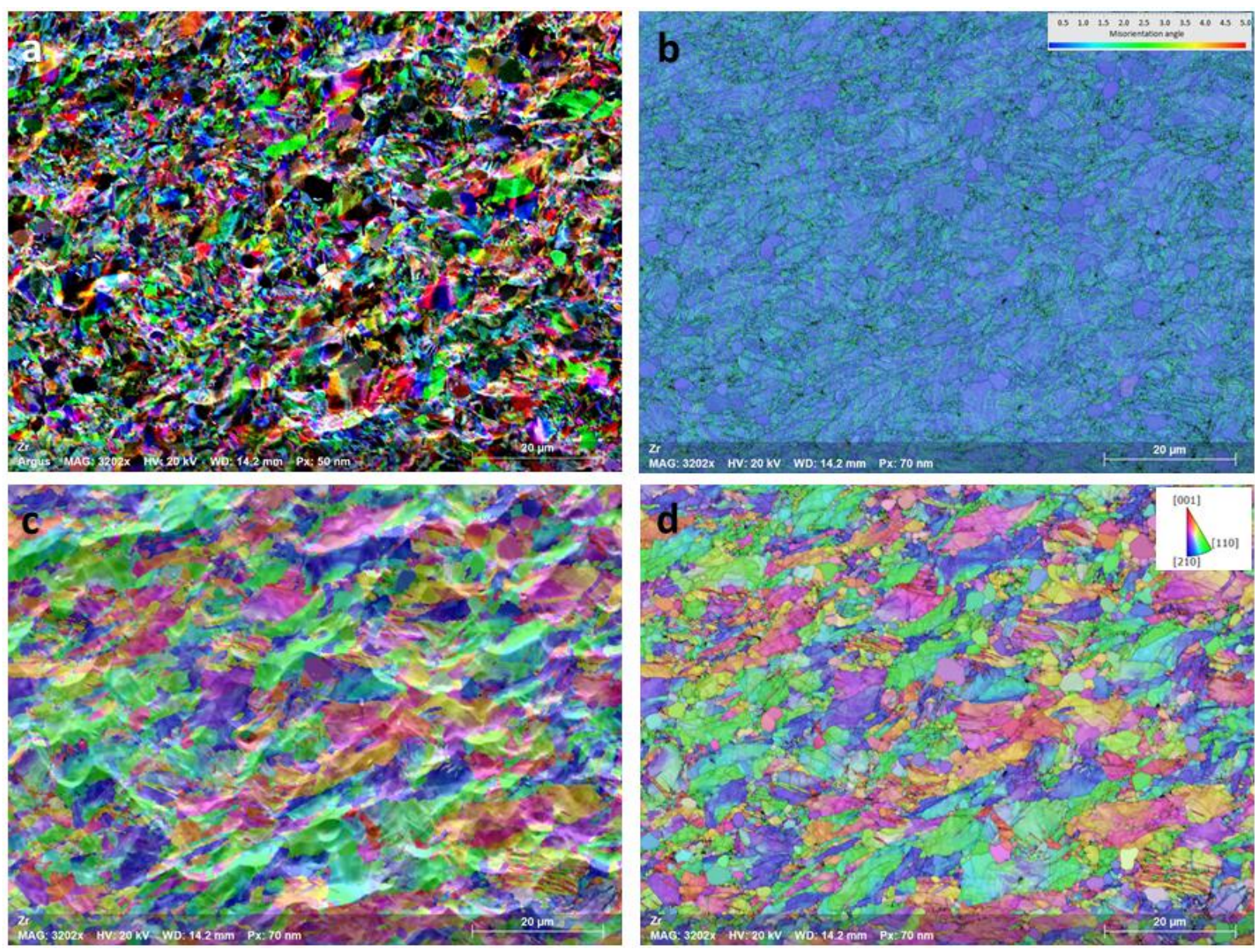

Figure 2. Example of broad ion beam milling effect on EBSD results: a) ARGUS ${ }^{\mathrm{TM}}$ color-coded orientation contrast image [Bruker Nano Analytics] of a bulk specimen of zirconium alloy b) corresponding kernel average misorientation (KAM) distribution in false color and pattern quality map as background, c) inverse pole figure (IPF) map overlaid on ARGUS ${ }^{\mathrm{TM}}$ color-coded orientation contrast image, $d$ ) inverse pole figure maps with pattern quality map as background. The KAM as well as IPF maps showing no correlation with the surface topography. EBSD data were acquired using e-FlashFS EBSD detector [Bruker Nano Analytics] with an indexing rate of $98 \%$.

\section{References}

[1] Kato, N. I. (2004). Reducing focused ion beam damage to transmission electron microscopy samples. Journal of Electron Microscopy, 53(5), 451-458. https://doi.org/10.1093/jmicro/dfh080

[2] Mayer, J., Giannuzzi, L. A., Kamino, T., \& Michael, J. (2007). TEM sample preparation and FIBinduced damage. MRS Bulletin, 32(5), 400-407. https://doi.org/10.1557/mrs2007.63

[3] Saowadee, N., Agersted, K., \& Bowen, J. (2012). Effects of focused ion beam Milling on electron backscatter diffraction patterns in strontium titanate and stabilized zirconia. Journal of Microscopy, 246(3), 279-286. https://doi.org/10.1111/j.1365-2818.2012.03616.x

[4] Nowakowski, P., Wiezorek, J., Spinelli, I., Ray, M., \& Fischione, P. (2019). Elastic and plastic strain measurement using electron backscatter diffraction technique: The influence of sample preparation. Microscopy and Microanalysis, 25(S2), 534-535. https://doi.org/10.1017/s1431927619003404 
[5] Nowakowski, P., Schlenker, J., Ray, M., \& Fischione, P. (2016). Sample preparation using broad argon ion beam Milling for electron backscatter diffraction (EBSD) analysis. Microscopy and Microanalysis, 22(S3), 12-13. https://doi.org/10.1017/s143192761600091x

[6] Giannuzzi, L., \& Michael, J. (2020). Ion-induced transformation of metastable phases. Microscopy and Microanalysis, 26(S2), 792-793. https://doi.org/10.1017/s1431927620015858

[7] Bonifacio, C., Nowakowski, P., Costello, K., Ray, M., Morrison, R., \& Fischione, P. (2019). Controlled environments from sample preparation to electron microscopy characterization. Microscopy and Microanalysis, 25(S2), 698-699. https://doi.org/10.1017/s1431927619004227

[8] Nowakowski P., Bonifacio C. S., Doux J., Meng S., Ray M. L., Fischione P. (2020, November). Gaining a better understanding of lithium ion battery functionality through scanning electron microscopy analytical studies [Conference session]. Materials Research Society 2020 Fall Meeting, Virtual, in press. 